\title{
COPING WITH THE DYNAMICS OF URBAN SUSTAINABILITY: POSITIONING URBAN EXPERIMENTATION PLATFORMS AS TOOLS FOR POLICY MAKING
}

\author{
S.-V. Rehm ${ }^{1, *}$, S. McLoughlin ${ }^{2}$, G. Maccani ${ }^{3}$ \\ ${ }^{1}$ EM Strasbourg Business School, Université de Strasbourg, HuManiS EA 7308, F-67000 Strasbourg, France; \\ sven.rehm@em-strasbourg.eu \\ ${ }^{2}$ Enable, Innovation Value Institute, School of Business, National University of Ireland Maynooth, W23 F2K8 Kildare, Ireland; \\ shane.mcloughlin@mu.ie \\ ${ }^{3}$ Ideas for Change, Ideas 3493 S.L., 08007 Barcelona, Spain; giovannimaccani@ideasforchange.com
}

KEY WORDS: Digital Platform, Collective Intelligence, System Dynamics, Urban Development, Urban Ecosystem, Urban Experimentation, Urban Governance, Urban Sustainability.

\begin{abstract}
:
This research considers the creation of resilient organizational models of urban governance to foster urban sustainability. Building on large-scale urban experimentation initiatives, we identify relevant factors in establishing urban experimentation with the help of digital platforms as a means to support such resilient organizational models. Our findings suggest that digital platforms help establish urban experimentation platforms (UXPs) as a core means to innovate towards urban sustainability. UXPs utilize digital platforms to coordinate policy measures and stimulate collective intelligence in order to reach a sustained local socio-technical transformation process. Based on these empirical findings, we use a systems lens to decompose urban experimentation into interconnected sub-systems and then describe some characteristics of their dynamic interaction. Our goal is to develop a System Dynamics model and simulations to support urban public authority decision makers in formulating and implementing policy interventions towards urban sustainability.
\end{abstract}

\section{INTRODUCTION}

Urban governance is confronted with challenges such as rising energy and water consumption, pollution, traffic and greenhouse gas emission and others more (Acuto and Parnell, 2016; United Nations, 2015; 2017). As a direct consequence, city municipalities determinedly engage in drafting and implementing policy agendas for improving urban life through sustainable development (Healey et al., 1999). The goal is to reach urban sustainability, understood as a city's capability to respond to societal challenges on a continued basis, and to proactively design the urban living environment in view of future societal and environmental good, on the local scale (Rehm et al., 2021).

Part of these efforts targets economic prosperity, increasingly a matter of city competitiveness, supported by creating attractive regulatory environments for businesses, people, culture and the environment (Visnjic et al., 2016; The Economist Intelligence Unit, 2013). In their city management, municipalities consequently pay particular attention to institutional conditions, place attractiveness, branding and identity, and strength of the local innovation ecosystem, as factors that various studies have identified to undergird city competitiveness (Khatoun and Zeadally, 2016; Visnjic et al., 2016; The Economist Intelligence Unit, 2013; van Winden et al., 2014).

Of particular importance is to understand how factors that contribute to city competitiveness can be sustained. To this end, municipalities need to find ways to attract, develop and retain capital, businesses and human talent, to engage in vivid exchange with other economic centres (van Winden et al., 2014). This latter point relates to the question of how citizens can be sustainably and collectively engaged as 'smart citizens', i.e., how citizen engagement, participation and empowerment can be continuously (re-) built and leveraged (Figueiredo Nascimento et al., 2016).

A prominent tool to support citizen engagement is to introduce urban experimentation, e.g., through Urban Living Labs, which foster the stimulation of grassroots urban innovation processesinvolving citizens, businesses, local research and other public institutions and further urban stakeholders - towards locally required solutions (Mukhtar-Landgren et al., 2019). This local or regional orientation in goals, means and stakeholder support is deemed important because it builds on locally available resources; a phenomenon identified as a decisive factor in the emergence of industrial clusters such as the Silicon Valley (Porter, 1990; Basole et al., 2018).

Several factors complicate attempts to provide generic governance responses to this question of sustainable urban development. The local and regional cultural, environmental and economic settings, legal boundary conditions and other factors more have to be reconciled recurrently with the conflicting demands and necessities for change, innovation and adaptation imposed by the heterogeneous weave of actors in the urban context and those beyond, and their divergent expectations and conceptions of rational progress (Figueiredo Nascimento et al., 2016). In essence, the question is how the recognition and reconciliation of the multiplicities of urban life can be collectively governed.

Current literature has thus framed the issue of urban sustainability as a complex, socio-technical transformation process that requires holistic approaches to urban management (Mora et al., 2019a; Miller et al., 2021; Rehm et al., 2021). Organization scholars are thus calling for identifying resilient organizational models of urban governance appropriate for the challenge of urban sustainability, and how these can be conceived and institutionalized by municipalities. To this end, our research investigates the organizational structures conditioning the dynamics of urban governance - that are instrumental to reaching urban sustainability. Particularly, we are interested in the various forms of organization and coordination of tasks, activities and information across organizational and decision-making structures; which help establish the collective capability for resiliency in the identifying, tackling and resolving of local issues through a continuous local innovation process. Our aim is to

\footnotetext{
* Corresponding author
} 
describe, model and simulate how the dynamics across these organizational structures and the urban innovation process unfolds.

In this short paper, we outline our perspective together with early empirical insights and results from system dynamics modelling that communicate our research in progress. In the following section 2, we first look at relevant aspects of urban experimentation, and outline our concept of urban experimentation platforms. In section 3 we describe our method and case studies. We highlight various aspects that speak to the decomposition of urban experimentation as a dynamic problem in section 4. Section 5 provides some conclusions and an outlook on future research.

\section{RELATED RESEARCH}

\subsection{Urban Experimentation Culture and Policy-making}

Urban experimentation (UX) extends the traditional approaches of urban planning. Its basic notion rests on the expectation that fostering local innovations can iteratively accelerate the transition towards sustainable and resilient cities (MukhtarLandgren et al., 2019). Given the multiplicities of today's urban (social) life, municipalities have become aware that innovative, dynamic and inclusive approaches are required as iterative steps towards sustainability. Thus, UX efforts tend to bring together various stakeholders with the capabilities for tackling societal challenges.

Given, the iterative nature of innovating towards urban sustainability, the challenge of UX lies in mirroring, foreseeing and responding to social, technological, environmental and further changes through continuous adaptations in policymaking. Adaptive policies thus need to advance over time in response to new information (Lempert and Groves, 2010). Various municipalities have taken on this challenge by adopting 'smart' agendas, with the aim to establish a culture of experimentation. Such UX culture continuously stimulates various stakeholders (including citizens) in collaborative innovation in order to facilitate more rapid, context-specific action for locally resilient and robust solutions.

From a global point of view, such local solutions are the outcome of a complex interplay of stakeholders across levels of policymaking: International, national, provincial, regional, local, and site planning policies and activities need to be reconciled (Wheeler, 2013). Thus, UX policy interventions need to weave together planning initiatives and institutions, and integrate and balance their efforts and interests (Wheeler, 2013; Mora et al., 2019b). Instituting and perpetually reassessing UX as part of policy-making and implementation, thus affords its consideration as an important form of urban governance.

One prominent vehicle to establish UX culture are urban laboratories, and more specifically, Urban Living Labs, which provide spaces and accessible drop-in centres for diverse stakeholders to co-create, experiment and innovate (MukhtarLandgren et al., 2019). If successful, they become condensation points for policy education and learning for citizens, training in innovation and transformative place-making and support the activation of experimentation partners (Mukhtar-Landgren et al., 2019; Wirth et al., 2019). Thus, when municipalities engage in policy-making and provide a fertile ground for labs to flourish, this can lead to entrepreneurial growth and narratives of impact, which in turn can reinforce broader diffusion mechanisms and strategies through which labs - and the related policies - create an even wider impact. This positive feedback loop has been characterized as one way to stimulate "systemic change" (Fuenfschilling et al., 2019).
Systemic change particularly hints at the role of urban business ecosystems (UBE). UBEs link the various urban stakeholder groups such as the government, utility providers, academia, entrepreneurs and all kinds of businesses and intermediaries (Visnjic et al., 2016, p. 116). As an organizational element and actor at play in UX, UBEs help to extend, connect and scale individual experimental initiatives to become part of a vivid urban innovation ecosystem, again positively feeding back to UX, i.e., contributing to sustain urban experimentation culture (Gascó, 2017; Rehm et al., 2021).

\subsection{Urban Experimentation Platforms (UXPs)}

The basic idea of Urban Experimentation Platforms (UXPs) is to link urban business ecosystems, urban experimentation with its related policy-making, and the locally instantiated collaborative innovation process (Rehm et al., 2021). While they have a digital platform at their core, they can be considered social assemblages, i.e., encompassing digital elements as well as associated organizational processes and policy measures (which can be constitutive as well as regulatory in nature) (see also Reuver et al., 2018, p. 126). UXPs are often managed by a public, often municipal authority, or respectively, by other types of organizations or consortia such as privately or University-led business incubators or transfer centres.

The key notion that accompanies UXPs is that as digital technology, they provide the principal capacity to orchestrate adaptive processes, aided by a growing reservoir of data, and thus helping implement adaptive and responsive policies (Rehm et al., 2021). This way, they represent an instrument or tool to enable more open, transparent and interactive forms of policy-making and policy implementation (more on openness can be found in Kornberger et al., 2017).

The role of data in this respect cannot be over-stressed. Here, UXPs principally act as data reference point for policy development (Rehm et al., 2021). They play a role as data aggregators or "observatories" enabling collection and provision of urban data, which makes these platforms possibly serve as critical drivers of research, technology transfer and commercialization (Miller et al., 2021; Rehm et al., 2021).

Despite numerous data-driven initiatives in the smart city context, various questions about UXPs are still unanswered with respect to how they can be resolved in the local context-given the specific conditions of the metropolitan region or city, the municipality and UBE stakeholders (Rehm and Faber, 2020). Such questions for instance comprise, which kind of data is to be collected, and which kind of analyses are required, how to use data to stimulate UBE stakeholder interactions, how to reach continuity in data management, how to leverage data observatories to foster citizen engagement, and what requirements for data driven services and their usage by stakeholders should be adopted, and others more. This issue also extends to the type of data to be collected, may it be geospatial data, data about existing public and private services, or 'simply' data about the UBE itself, e.g., the number of firms in a specific sector, and their intentions and capacities to engage in urban experimentation (Rehm et al., 2017; Rehm and Faber, 2020). We are also lacking long-term studies of UXPs, their underlying business models, apps, and their interplay with supportive stakeholders that help institutionalize UX culture and establishing related policies.

\section{METHOD}

In our research, we draw on insights from several large-scale smart city initiatives comprising multiple concluded case studies to which the authors were involved as researchers. Each of these 
case studies featured specific problem settings and boundary conditions for urban experimentation and it involved building, implementing and iteratively refining digital tools for the specific urban contexts in the case studies. The authors' experiences during the case studies thus provide a rich reserve of insights for discussing the dynamics involved to urban experimentation initiatives. Particularly, we relate to results achieved in the EU Horizon 2020-supported collaborative research projects OrganiCity and Making Sense. Our research question considers, how the dynamics of urban experimentation with respect to achieving resiliency of the local innovation process unfolds? We draw on the empirical case base to model and simulate the dynamics of urban experimentation with the help of System Dynamics (Sterman, 2000). Such models allow to capture and discuss the dynamics inherent to the models and identify relevant systemic patterns and related policy recommendations to inform policy makers. ('Policy recommendations' as used in System Dynamics jargon relate to the management responses, decisions and interventions of stakeholders as suggested by a considered system model and simulations, which in our case can refer to urban policy-making, but goes beyond that).

\subsection{System Dynamics for Modelling Urban Experimentation}

We used System Dynamics (SD) as a lens and language to investigate the dynamics of UX because our aim is to "analytically capture the formatting of policy into practice," an objective seen as imperative when engaging with UX (Kornberger et al., 2017). Particularly, SD allows studying how municipalities and UBE stakeholders mutually re-constitute and reconfigure policies, technology and organization (a task postulated by Kornberger et al., 2017). This resonates with the intention of SD "(aiming) to change the mental models that people use to represent the real world. For this to happen, individuals must be sufficiently involved in the modeling process to internalize lessons about dynamic feedback behavior" (Forrester, 1995, p. 14).

Our research started from examining the decision-making hierarchy that connects policy-making with urban experimentation. Through our engagement in the case studies and participant observation, we inference with and reflect on the mental models of UX stakeholders and decision-makers as prescribed for SD (Luna-Reyes and Andersen, 2003). A first result of this work is a policy structure diagram for UX. Our upcoming work targets the SD modelling cycle and simulation completion, using data from real policy-making decision processes.

\section{DECOMPOSITION OF URBAN EXPERIMENTATION}

\subsection{Dynamic Hypothesis on Urban Experimentation}

System Dynamics starts with the formulation of the problem to be considered, and the formulation of a dynamic hypothesis explaining the problem's dynamics as endogenous consequences of a feedback structure (Sterman, 2000). As we argue, urban experimentation necessitates such a feedback structure when UX is to be sustained towards urban sustainability. This feedback structure unfolds between the involved urban stakeholders, their decisions and other relevant factors and variables; all intending to boost a successful local innovation process able to respond to emerging social challenges. Our dynamic hypothesis builds on the distinct role of UXPs as "actors" in the UX setting, allowing to stimulate and manage innovations and policies, and to extend policy decisions across various levels.

\subsection{Policy Structure Diagram for UX}

We decompose our problem setting into several loops seen as critical for UX dynamics (Morecroft, 1982). These loops are delimited by sub-systems of the overall problem, which can be determined by identifying areas with coherent goals, decisionmaking processes, or mechanisms (indicated in Figure 1).

In our context, the subsystems are (1) the urban business ecosystem (UBE) with its own mechanisms for ecosystem governance for stakeholders' motivation to participate in UX or in lobbying for social change; (2) the set of UX related policies, publicly provisioned resources, funding agencies and its power to issue statutory instruments that represent the nucleus of all UX endeavours; and (3) the UX instantiation of projects and initiatives, with their needs and outcomes, as the operational level of UX, directly linked with the local innovation process.

A further notable element is (4) the UXP as evolving tool available as public platform, i.e., involving diverse stakeholder groups. The UXP represents a subsystem, as it invites contributions, such as apps for innovation project management developed by experimenters, and as it directly influences all other three subsystems, through provision of apps and services to experimenters, by making urban data accessible (observatory function) and also by supporting implementation of UX related policies.

Figure 1 shows subsystems and connecting loops as a policy structure diagram. Loops 1 and 2 represent commonly assumed feedback in UX policy-making, e.g., between policies that fund urban labs (loop 1), or those that foster a cooperative culture in the UBE (loop 2). Loops 3 to 5 indicate how novel digital capabilities through UXPs alter UX dynamics, e.g., if analysis of data with help of the UXP shows which experimentation initiatives are most successful, policies can be adapted accordingly (loop 3). Such data-driven insights might be transferable to policies themselves (loop 5) and to the UBE (loop 4). In general, UXPs act on all levels of UX policy-making, informing UBE stakeholders as observatory, allowing for UX policy adaptation and supporting UX experimentation.

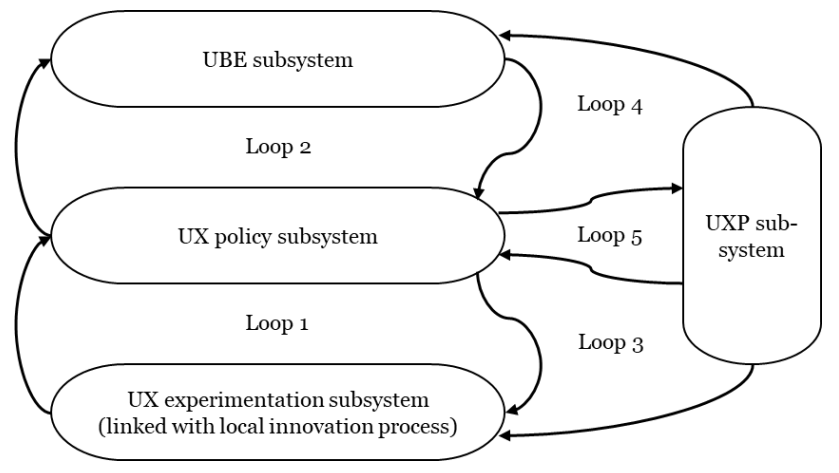

Figure 1. Policy structure diagram as decomposition of UX.

\subsection{UXPs' Digital Capabilities for Urban Experimentation}

UXPs feature adaptable digital services and related functionalities to support implementation of policy via experiments to innovate, and to inform and stimulate UX policy adaption. For each subsystem, various digital services and related functionalities have been implemented in the UXP OrganiCity project. Examples include, for the UBE subsystem: APIs to enable open data access and sharing, stimulating experimenters' and other UBE stakeholder leveraging of open data; or the Urban Data Observatory, to provide visibility to UBE stakeholders to help them engage with local experiments and urban data. For the 
UX policy subsystem, for instance, a Management Portal to integrate UX projects was prepared; and scenario tools for urban challenge formulation and voting were realized. The Urban Data Observatory also can also act as a portal of data about the city for policy makers. The UX experimentation subsystem was provided with portals for experimenters and communities, and with an assets directory that federated all the data assets within the UXP, allowing discovery, access and sharing of urban data. These are just some of the functionalities implemented in our case study; many more are conceivable.

\subsection{UXPs in a Vision of Collective Intelligence for Urban Experimentation}

Our case study and early decomposition indicate that, with their capacity as reference points of urban data, information and knowledge, UXPs can successfully act as open digital platforms that provide (digital) services for urban experimentation to various stakeholders. The stakeholders ground their decisions on shared data and analyses, and cooperate along shared services. This way, UXPs become a tool to enable a collective capability that emerges from - and reconditions, or regenerates, itselffrom the interaction of citizens, UBE stakeholders and municipalities as policy-makers. This collective capability dynamically leverages collective intelligence, existing as evolving set of knowledge, resources and mediating (digital and other) tools and policies towards a collective performance measured as progress towards urban sustainability that may potentially subsist in collective action as resilient organizational model of urban governance.

The core challenge of collective action lies not in facilitating a single innovation process, but to enable repeated and overlapping phases of (a) collective ideation of experiments, (b) collaborative, open innovation through dedicated urban initiatives, and (c) reconsideration of how UX results and changing conditions necessitate novel policies, policy adaption or experimentation. It specifically resonates with issues of attracting, integrating and retaining (where necessary) relevant citizens and other urban stakeholders as a diverse and heterogeneous set of agents across these phases.

Such integration, for example, while well-researched for open innovation projects, crowd-based online communities and stable contexts etc., is unique to our context as it needs to be maintained and transitioned across the abovementioned phases of ideation, innovation and policy adaption; and in different configurations of actors, settings and means. For instance, the output of local experiments requires collective quality assessment, and likewise broad discussion and reception across the diversity of citizens, interest groups and communities in the urban setting, who are directly and indirectly affected. Another example for collective action are knowledge processes that need to be facilitated between actors or at least stimulated, through aggregation of information and data, and by providing distinct services for UX and implementation of solutions. In this respect, our past research has indicated for instance that visualizations of urban data and particularly UBEs, can support identifying regional coverage of offered services, or uncovered service demands, which might allow quantifying citizen requests for better service coverage or stimulating innovation (Rehm et al., 2017).

We conjecture that in discussing how collective action can be fostered and sustained, environmental complexity as well as further distinctive contextual factors need to be considered, as otherwise for instance local solutions or positive notions of change might be lost and thus collective action not sustained. From our early modelling we also conjecture that UXPs can essentially contribute to a vision of Collective Intelligence for urban experimentation.

\subsection{Characterization of Policy Interventions in UX and Stimulation of Collective Intelligence}

The digital services provided by UXPs are the means at hand for implementing UX policies, for building a municipality's capability for UX, and for sustaining its momentum through collective action. The services produce, collect and in some cases analyse data and information, making it accessible to UX stakeholders and the UBE. They also help in coordinating processes and enable, support and/or enhance citizen engagement in experimentation; and they principally allow to leverage local resources and knowledge.

However, this principle capacity raises the question how exactly policy interventions become effective in establishing continued engagement and collaboration of UBE stakeholders, and which factors govern the related dynamics. In particular, there is a gap in understanding which are the dynamic regulating forces that govern how the collective actions across the phases mentioned above become effective. Our future goal is to determine these 'forces,' because they undergird any capability for resiliency, and ultimately fuel the continuous socio-technical transformation process. We conceive them as the cybernetic phenomena expressed in informational, coordination, decision and control structures that elucidate the dynamics between subsystems as conceived above.

There are some candidates for conditions bringing out such forces that we have so far identified and which we will investigate in our future research. Among them are, questions of control, autonomy, and freedom; issues of enabling or restricting choice; aspects of adaptability, robustness, viability, variety, spatial and temporal synchronization, diversity, complexity, and others.

We hope that a better characterization of policy interventions by drawing on these forces will help improve our picture and understanding of UX, and help leverage collective intelligence for the common good, on a local level.

\section{CONCLUSIONS AND FUTURE RESEARCH}

Our early findings from case studies indicate that municipalities need to develop novel capabilities in order to appropriately respond to the timely and escalating challenge of urban sustainability. These capabilities centre around the establishment of an urban experimentation culture that fosters facilitation of urban ecosystem governance, the empowerment of citizen cocreation, and the safeguarding of local innovation.

In order to prepare the ground for a description of the dynamics inherent to urban experimentation, we decompose this task as decision-making structure. This structure comprises the governance of urban business ecosystems, the installation of urban experimentation as part of municipal policy-making, the implementation of urban experimentation as operative process, and the installation of an open urban experimentation platform. The primary challenge in establishing a resilient organizational model for urban experimentation lies in sustaining experiments, and learning from experiments in such a way as to produce relevant but discrete innovation outcomes, while stimulating a continuous socio-technical transformation process. In this sense, discrete experiments are pacing local innovations on an operative - and transformations on a higher organizational level, which necessitates regular adaptation of urban policies and stimulations of the surrounding urban ecosystems.

Our modelling exercise allowed us to take first steps in formulating urban sustainability as a dynamic problem: How can urban governance sustain the structural adaptation of urban experimentation under the premise of dynamic partnerships, 
adaptive policy, and changing requirements for sustainability (i.e., new challenges to solve local problems)?

With the help of System Dynamics, we have begun a modelling process aiming at running simulation models, which will allow us to consider and discuss local urban boundary conditions involving local resources and policies, initiatives, culture and experimenters. The idea is to formulate 'policy interventions,' i.e., guidelines for sustaining collective action, as recommendations to urban public authority decision makers. The contribution of this short paper is to establish key concepts and considerations, and to report on the progress of our research and the foundational aspects of establishing a System Dynamics model for urban sustainability.

\section{ACKNOWLEDGEMENTS}

The OrganiCity project was co-funded by the European Commission under contract H2020-645198 and the Making Sense project under contract H2020-688620. This work was supported with funding from Science Foundation Ireland grant 13/RC/2094 and Intel Labs Europe.

\section{REFERENCES}

Acuto, M., Parnell, S., 2016: Leave no city behind. Science, 352 , 873.

Basole, R. C., Park, H., Chao, R. O., 2018: Visual Analysis of Venture Similarity in Entrepreneurial Ecosystems. IEEE Trans. Eng. Manage., 1-15. doi.org/10.1109/TEM.2018.2855435.

Figueiredo Nascimento, S., Cuccillato, E., Schade, S., Guimarães Pereira, A., 2016: Citizen Engagement in Science and PolicyMaking, EUR 28328 EN. doi.org/10.2788/40563.

Fuenfschilling, L., Frantzeskaki, N., Coenen, L., 2019: Urban experimentation \& sustainability transitions. European Planning Studies, 27, 219-228.

Forrester, J. W., 1995: The beginning of system dynamics. McKinsey Quarterly, 4, 4-16.

Gascó, M., 2017: Living labs: Implementing open innovation in the public sector. Government Information Quarterly, 34, 90-98.

Healey, P., Khakee, A., Motte, A., Needham, B., 1999: European developments in strategic spatial planning. European Planning Studies, 7, 339-355, doi.org/10.1080/09654319908720522.

Khatoun, R., Zeadally, S., 2016: Smart Cities: Concepts, Architectures, Research Opportunities. Communications of the ACM , 59, 46-57. doi.org/10.1145/2858789.

Kornberger, M., Meyer, R., Brandtner, C., Höllerer, M., 2017: When Bureaucracy Meets the Crowd: Studying Open Government in the Vienna City Administration. Organization Studies, 38, 179-200.

Lempert, R. J., Groves, D. G., 2010: Identifying and evaluating robust adaptive policy responses to climate change for water management agencies in the American west. Technological Forecasting and Social Change, 77, 960-974.

Luna-Reyes, L. F., Andersen, D. L., 2003: Collecting and analyzing qualitative data for system dynamics: methods and models. System Dynamics Review, 19, 271-296.
Miller, H. J., Clifton, K., Akar, G., Tufte, K., Gopalakrishnan, S., MacArthur, J., Irwin, E., 2021: Urban Sustainability Observatories: Leveraging Urban Experimentation for Sustainability Science and Policy. Harvard Data Science Review. doi.org/10.1162/99608f92.2025202b.

Mora, L., Deakin, M. Reid, A., 2019a: Combining co-citation clustering and text-based analysis to reveal the main development paths of smart cities. Technological Forecasting and Social Change, 142, 56-69. doi.org/10.1016/j.techfore.2018.07.019.

Mora, L., Deakin, M., Reid, A., 2019b: Strategic principles for smart city development: A multiple case study analysis of European best practices. Technological Forecasting and Social Change, 142, 70-97.

Morecroft, J. D., 1982: A critical review of diagramming tools for conceptualizing feedback system models. Dynamica, 8, 20 29.

Mukhtar-Landgren, D., Kronsell, A., Voytenko Palgan, Y., von Wirth, T., 2019: Municipalities as enablers in urban experimentation. Journal of Environmental Policy \& Planning, 21, 718-733. doi.org/10.1080/1523908X.2019.1672525.

Porter, M. E., 1990: The competitive advantage of nations, Free Press.

Rehm, S.-V., Faber, A., 2020: Building the City's Business Networks: Using Visualisations for Business Ecosystem Governance. In P. Planing, P. Müller, P. Dehdari, T. Bäumer (Eds.), Creating Innovations for Metropolitan Areas: Intelligent Solutions for Infrastructure, Mobility, and Applications for Citizens, 243-254, Springer.

Rehm, S.-V., Faber, A., Goel, L. 2017: Visualizing Platform Hubs of Smart City Mobility Business Ecosystems. Proceedings of the 38th International Conference on Information Systems, South Korea 2017. https://aisel.aisnet.org/icis2017/ DigitalPlatforms/Presentations/14/

Rehm, S.-V., $\quad$ McLoughlin, S., $\quad$ Maccani, G., 2021 : Experimentation Platforms as Bridges to Urban Sustainability. Smart Cities, 4, 569-587, doi.org/10.3390/smartcities4020030.

Reuver, M. de, Sørensen, C., Basole, R. C., 2018: The digital platform: a research agenda. J Inf Technol., 33, 124-135.

Sterman, J. D., 2000: Business dynamics: Systems thinking and modeling for a complex world, Irwin/McGraw-Hill.

The Economist Intelligence Unit, 2013: Hotspots 2025: Benchmarking the Future Competitiveness of Cities. citigroup.com/citi/citiforcities/pdfs/hotspots2025.pdf (26 April 2021).

United Nations, 2015: Transforming Our World: The 2030 Agenda for Sustainable Development. A/RES/70/1. United Nations. sdgs.un.org/2030agenda (31 March 2021).

United Nations, 2017: New Urban Agenda. A/RES/71/256. United Nations. habitat3.org/wp-content/uploads/NUAEnglish.pdf (31 March 2021).

Wheeler, S. M., 2013: Planning for Sustainability: Creating Livable, Equitable and Ecological Communities, Taylor and Francis. 
Winden, W. van, Braun, E., Otgaar, A., Witte, J.-J., 2014: Urban innovation systems: What makes them tick?, Routledge.

Wirth, T. von, Fuenfschilling, L., Frantzeskaki, N., Coenen, L., 2019: Impacts of urban living labs on sustainability transitions: mechanisms and strategies for systemic change through experimentation. European Planning Studies, 27, 229-257.

Visnjic, I., Neely, A., Cennamo, C., Visnjic, N., 2016: Governing the City: Unleashing value from the business ecosystem. California Management Review, 59, 109-140, doi.org/10.1177/0008125616683955. 